\title{
Wavelet and SVD smoothing for Pole Figures of Titanium
}

\author{
T.M. Ivanova, T.I. Savyolova \\ Moscow Engineering Physics Institute, Kashirskoe shosse 31, 115409 Moscow, Russia \\ akmenukas@comtv.ru
}

Diffraction Pole density data are affected by many factors such as texture inhomogeneity, insufficient statistics, instrumental errors etc. As a result we have Pole figures (Pfs) containing data noised by an irregular component. In some cases this component may be rather strong and exert heavy influence on the modeling process making the model unstable. Thus Pfs data need to be smoothed by some methods.

In this work we try to interpret Pole figure data of Titanium as images and to treat the Pfs with some widespread methods of image processing. Namely we use singular value decomposition (SVD) smoothing ${ }^{1}$ and a simplified variant of wavelet data processing ${ }^{2}$. These methods both base on the orthogonal factorizations of the initial data.

\section{SVD smoothing}

Usually Pf values are presented by rectangular matrices $(m \times n)$. These matrices can be factorized as

$$
P=U S V^{T},
$$

where $U$ and $V$ are orthogonal matrices $(\mathrm{m} \times \mathrm{m})$ and $(\mathrm{n} \times \mathrm{n})$ correspondingly. $S$ is $(\mathrm{m} \times \mathrm{n})$ diagonal matrix containing singular values. The singular values can be ranked so that

$$
\sigma_{1} \geq \ldots \geq \sigma_{n} \geq 0
$$

Then another possible presentation of the Pf data is a sum of external products of $U$-columns by $V^{T}$-rows

$$
P=\sum_{k=1}^{n} \sigma_{k} \mathbf{u}_{k} \mathbf{v}_{k}^{T}
$$

The bigger value of $\sigma_{k}$ the greater contribution of corresponding summand. Small singular values are responsible for high frequency components of the data, so for smoothing we save some first components of the sum with the biggest values of $\sigma$. Dynamic range of singular values for Pfs of Titanium is represented in Fig.1.

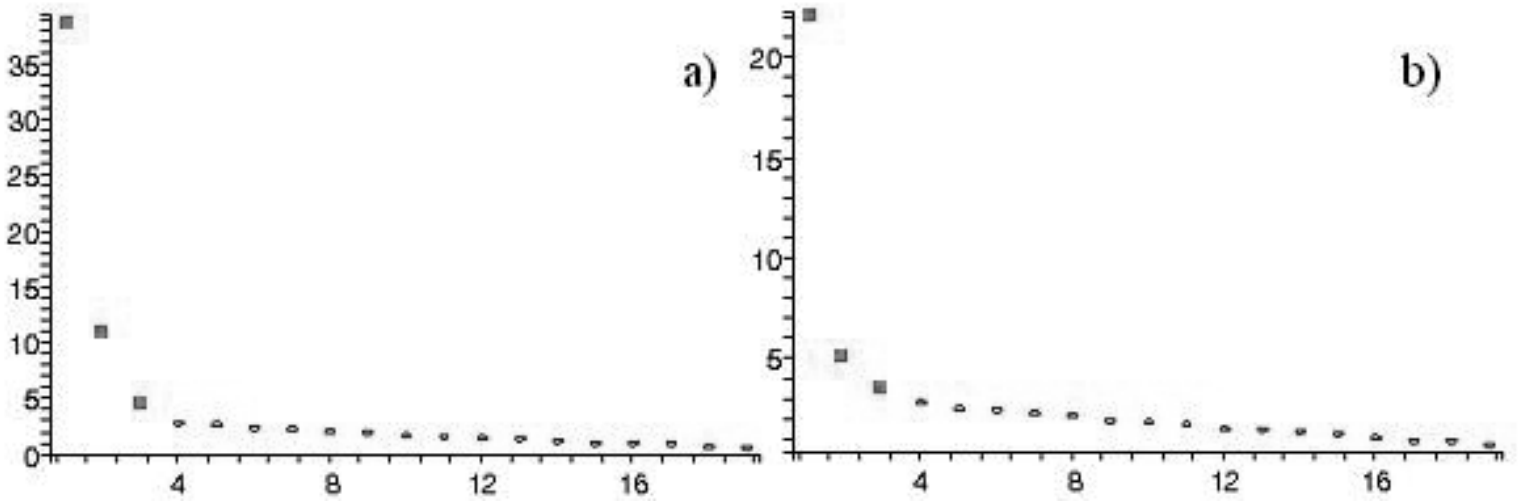

Fig.1. Dynamic range of singular values. a) For Pf $\{0002\}$ of Titanium. $\sigma_{1}=38.6, \sigma_{2}=10.5, \sigma_{3}=3.9$, others less than 2.8. b) For Pf $\{10 \overline{1} 0\}$ of Titanium. $\sigma_{1}=21.8, \sigma_{2}=5.0, \sigma_{3}=3.2$, others less than 2.6.

The singular values decrease very rapidly. It allows to compress diffraction information strongly. 
The main features of texture remain observed even in the case we keep only one singular number (Figs. 2b, 3b). In the case we keep three singular numbers Pf can be recovered very well, although somewhat smoothed (see Figs. 2c, 3c). This fact allows to estimate effective number of parameters in the mathematical model explaining experimental data.

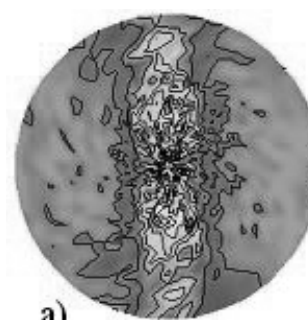

a)
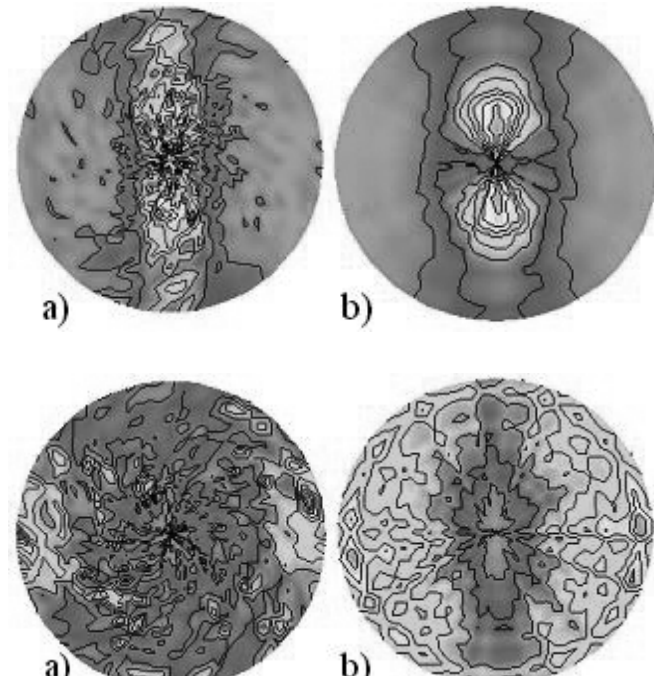

b)

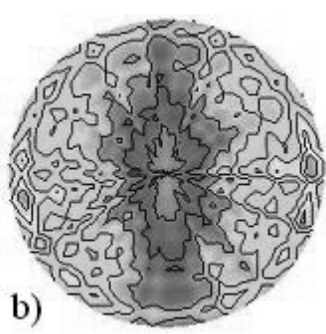

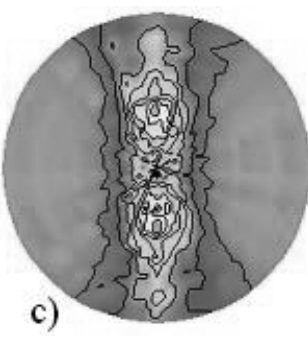

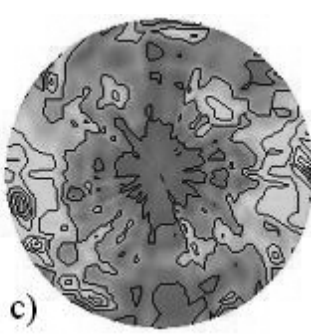

Fig.2. a) Initial Pf $\{0002\}$ of Titanium. $P_{\max }=3.1, P_{\min }=0.0$. b) Only one singular number $\sigma_{1}=38.6$ is used. $P_{\max }=$ $2.5, P_{\min }=0.2$. c) Three greatest singular values $\sigma_{1}=38.6, \sigma_{2}=10.5, \sigma_{3}=3.9$ are used (others less than 2.8). $P_{\max }=$ $3.0, P_{\min }=0.1$.

Fig.3. a) Initial Pf $\{10 \overline{1} 0\}$ of Titanium. $P_{\max }=2.6, P_{\min }=0.0$. b) Only one singular number $\sigma_{1}=38.6$ is used. $P_{\max }=$ $1.4, P_{\min }=0.1$. c) Three greatest singular values $\sigma_{1}=21.8, \sigma_{2}=5.0, \sigma_{3}=3.2$ are used (others less than 2.6). $P_{\max }=2.5$, $P_{\text {min }}=0.1$.

\section{Wavelet smoothing}

We apply a variant of discrete wavelet transform (DWT) using wavelets with 4 coefficients (Daubechies 4) $)^{3}$. DWT needs the data to be represented on the mesh $2^{k} \times 2^{l}$. To treat the Pf as a simple plane image $(128 \times 128)$, further denoted as $P_{\text {plane }}$, we performed bilinear resampling of extended stereographic projection of the initial data. The wavelet decomposition of the resampled data has a form

$$
W=D P_{\text {plane }} D^{T},
$$

where $D$ is the orthogonal matrix of DWT. While $P_{\text {plane }}$ represents the data in the initial basis, $W$ represents them in another one. The elements of $W$ has wider dynamic range than $P_{\text {plane }}$ data, but most of its elements have close to zero values (see Fig. 4).
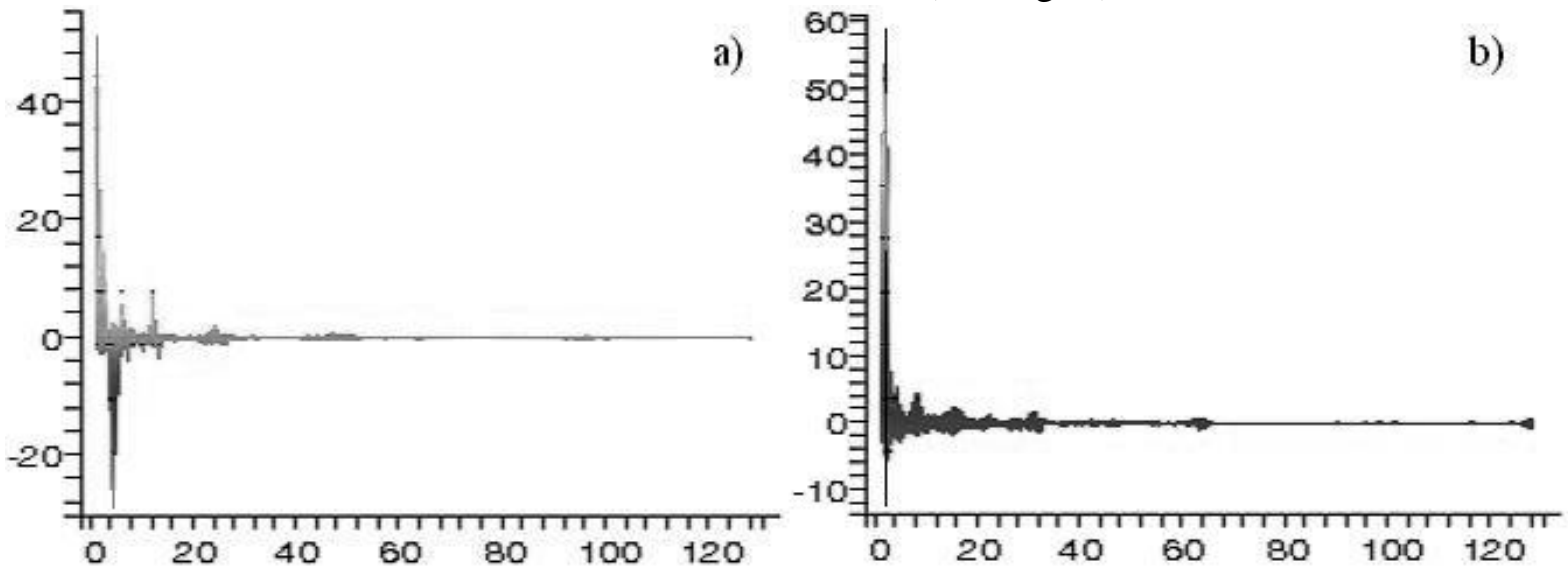

Fig.4. Dynamic range of wavelet coefficients (Profile). a) For Pf $\{0002\}$ of Titanium, $W_{\max }=53.6, W_{\min }=$ -28.8 . b) For Pf $\{10 \overline{1} 0\}$ of Titanium, $W_{\max }=59.4, W_{\min }=-12.3$. 
After some processing procedure we have a set of coefficients $\tilde{W}$. Since DWT is orthogonal, for inverse transform we get

$$
\tilde{P}_{\text {plane }}=D^{T} \tilde{W} D \text {. }
$$

There are several variants of data smoothing. Frequency thresholding consist in zeroing of high frequency elements of $W$ ( see Figs. 5e, 5f, 6e, 6f ). For less expressed blurring we have multiplied high frequency coefficient by some factor $\mu<1$ (see Figs. 5b, 5c, 6b, 6c ). Hard thresholding implies set to zero elements with absolute values less than some threshold value $\lambda$ ( see Figs. 5d, 6d), whereas soft thresholding is given by the relation

$$
\tilde{W}_{i j}(\lambda)=\left(W_{i j}-\operatorname{sign}\left(W_{i j}\right) \cdot \lambda\right) \cdot \theta\left(\left|W_{i j}\right|-\lambda\right) .
$$

Soft smoothed Pfs are represented in Figs. 5g, 6g. The subband of zeroing, the factor $\mu$ and the threshold value $\lambda$ are the parameters which may be varied.

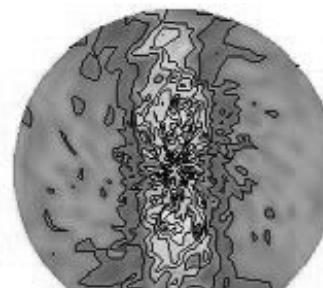

a)

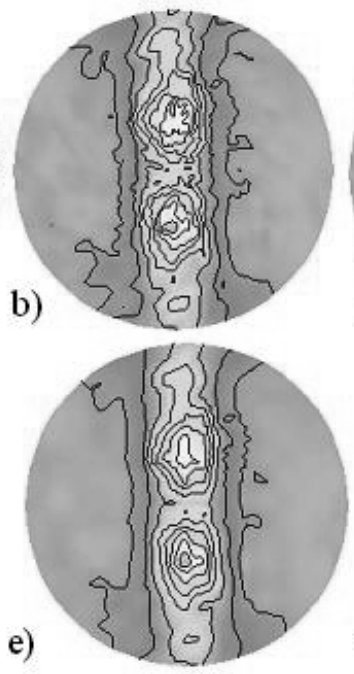

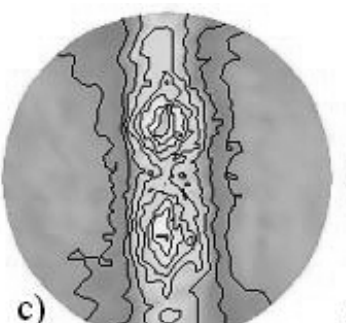

c)

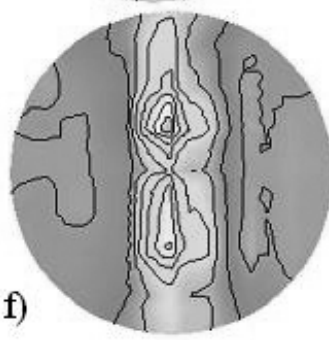

d)
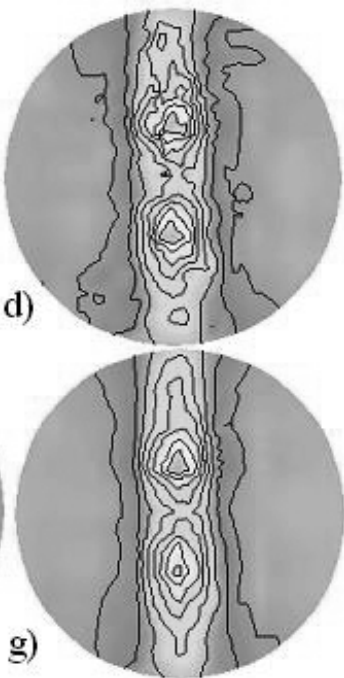

Fig.5. a) Initial Pf $\{0002\}$ of Titanium. $P_{\max }=3.1, P_{\min }=0.0$. b) $63 / 64$ of wavelet coefficients are multiplied by factor $\mu=0.5$. $P_{\max }=3.0, P_{\min }=0.1$. c) $255 / 256$ of wavelet coefficients are multiplied by factor $\mu=0.5$. $P_{\max }=2.8, P_{\min }=0.1$. d) Hard thresholding. Threshold level $\lambda=1$.0. All coefficients with absolute value less than $\lambda$ are zeroed. $P_{\max }=2.7, P_{\min }=0.1$. e) $63 / 64$ of wavelet coefficients are zeroed. $P_{\max }=2.9$, $P_{\min }=0.2$. f) $255 / 256$ of wavelet coefficients are zeroed. $P_{\max }=2.9, P_{\min }=-0.1$ g) Soft thresholding. Threshold level $\lambda=1$.0. $P_{\max }=2.6, P_{\min }=0.0$.

\section{Discussion of results}

Presented Pole figures of Titanium contain rather strong irregular component. It is hard to reveal actual texture components directly from raw data. After SVD smoothing the main parameters of the components such as peak position and approximate width became more clear, but the degree of smoothness is not sufficient yet (especially for Pf $\{10 \overline{1} 0\}$.)

The main part of great wavelet coefficients is located in low frequency subband. Coefficients in higher frequency subband must be decreased or zeroed for smoothing. Figures $5 \mathrm{f}$ and $6 \mathrm{f} \mathrm{look}$ somewhat oversmoothed, but processes $5 \mathrm{c}, 5 \mathrm{~d}, 5 \mathrm{e}, 6 \mathrm{c}, 6 \mathrm{~d}, 6 \mathrm{e}$, and especially soft thresholding $5 \mathrm{~g}$ and $6 \mathrm{~g}$ seem to be very appropriate. 

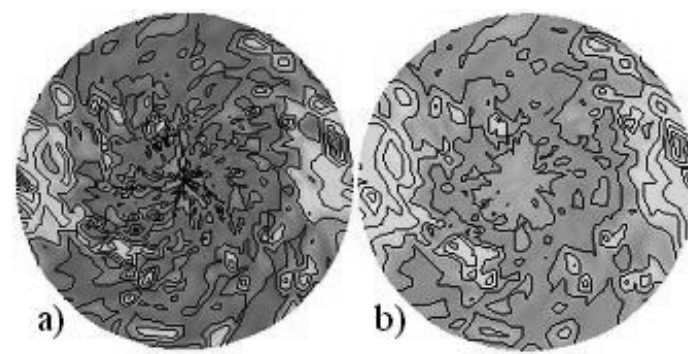

b)
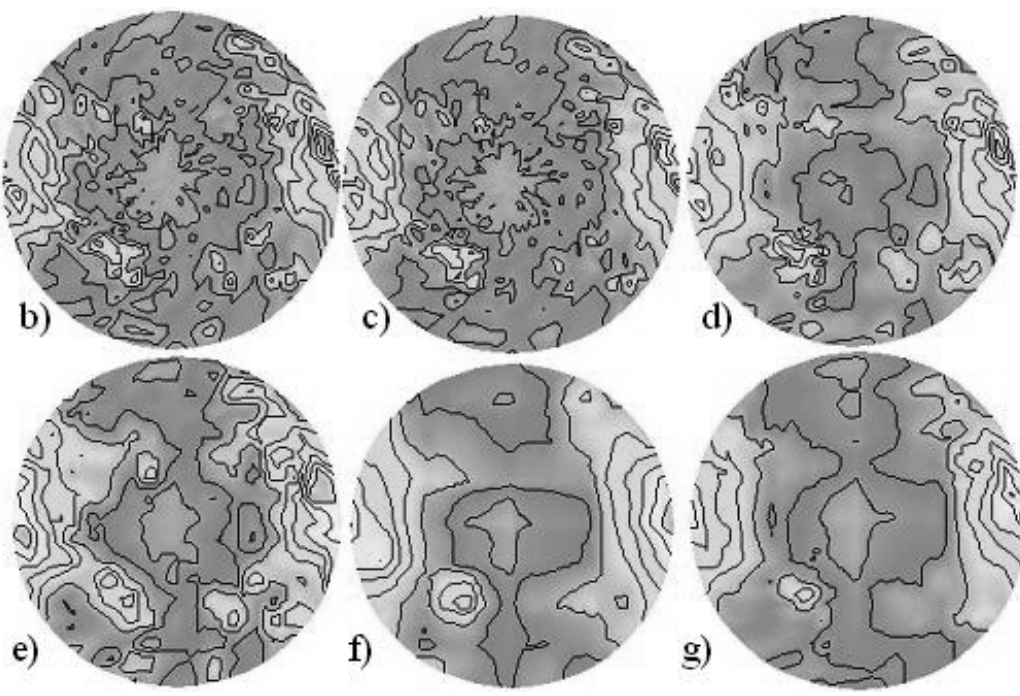

d)

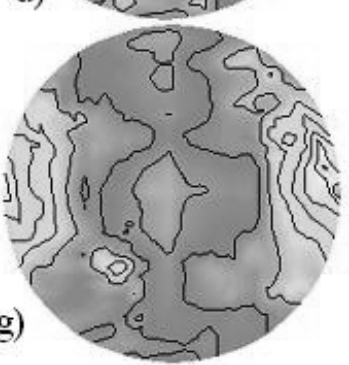

Fig.6. a) Initial $\operatorname{Pf}\{10 \overline{10}\}$ of Titanium. $P_{\max }=2.6, P_{\min }=0.0$. b) $63 / 64$ of wavelet coefficients are multiplied by factor $\mu=0.5$. $P_{\max }=2.2, P_{\min }=0.3$. c) $255 / 256$ of wavelet coefficients are multiplied by factor $\mu=0.5$. $P_{\max }=2.1, P_{\min }=0.2$. d) Hard thresholding. Threshold level $\lambda=1$.0. All coefficients with absolute value less than $\lambda$ are zeroed. $P_{\max }=2.1, P_{\min }=0.1$. e) $63 / 64$ of wavelet coefficients are zeroed. $P_{\max }=1.7$, $P_{\min }=0.2$. f) $255 / 256$ of wavelet coefficients are zeroed. $P_{\max }=1.7, P_{\min }=0.1$. g) Soft thresholding. Threshold level $\lambda=1$.0. $P_{\max }=1.8, P_{\min }=0.2$.

\section{Conclusion}

SVD smoothing is very simple procedure, but it does not possess powerful flexibility. We can only perform hard thresholding, i.e. simple zeroing of small singular values. Wavelet smoothing is much more flexible. It allows hard and soft thresholding as well as controlled blurring. Nevertheless SVD smoothing is very useful procedure allowing to estimate amount of model parameters for Pf data representation. Combination of these two methods seems to be advanced.

\section{Acknowledgements}

This work is supported by the State Contract 02.513.11.3340.

1. D. Kahaner, C. Moler, S. Nash, Numerical Methods and Software. Englewood Cliffs, NJ: P rentice Hall (1989), $575 \mathrm{pp}$.

2. S. Welstead, Fractal and Wavelet Image Compression Techniques. SPIE Optical Engineering Press (2002), $320 \mathrm{pp}$.

3. I. Daubechies, Ten Lectures on Wavelets. Society for Industrial and Applied Mathematics (1992), 461 pp. 